\title{
WAVELENGTH-SELECTIVE LIGHT QUENCHING OF BIOCHEMICAL FLUOROPHORES
}

\author{
Ignacy Gryczynski, Józef Kuśba, ${ }^{*}$ and Joseph R. Lakowicz \\ University of Maryland School of Medicine, Center for Fluorescence Spectroscopy; Medical \\ Biotechnology Center, Department of Biochemistry and Molecular Biology, 725 West Lombard Street, \\ Baltimore, Maryland 21201 \\ (Paper JBO-030 received Aug. 10, 1995; revised manuscript received June 12, 1996; accepted for publication June 18, 1996.)
}

\begin{abstract}
The use of light quenching to selectively eliminate the emission of biochemical fluorophores based on the emission wavelength is described. To demonstrate the possibility of wavelength-selective light quenching, a mixture of two fluorophores, 4-(dimethylamino)-4'-cyanostillene (DCS) and Prodan, emitting at different wavelengths was examined first. The emission spectrum and intensity decay were altered by the 570-nm quenching pulse due to selective quenching of the longer wavelength emission of DCS. Quenching of the solvent-sensitive fluorophore partially bound to human serum albumin and partially in the aqueous phase was then examined. Light quenching with a long wavelength $(570 \mathrm{~nm})$ time-delayed pulse selectively quenched the Prodan fluorophore in the aqueous phase while in the presence of the Prodan bound to human serum albumin, which emitted at shorter wavelengths, was not quenched. Using one-beam short wavelength excitation and quenching, a selective quenching of the blue-shifted emission of ribonuclease $T_{1}$ in the presence of the red-shifted emission of the tryptophan residue in adrenocorticotropic hormone was observed. In both systems wavelength-selective light quenching was demonstrated by a shift in the emission spectra, and by changes in the intensity decay consistent with preferential quenching of one species. Light quenching is instantly reversible by blocking or defocusing of the quenching beam. It can occur for inaccessible residues or in viscous solvents, and thus can be of wide applicability for resolving the complex emission of biological macromolecules. () 1997 Society of Photo-Optical Instrumentation Engineers.
\end{abstract}

Keywords wavelength-selective light quenching, time-resolved fluorescence, biophysics.

\section{INTRODUCTION}

Quenching of fluorescence is widely used in biochemical research as a method for studying the structure and dynamics of macromolecules. ${ }^{1}$ A wide variety of substances are known to act as collisional quenchers, including oxygen, iodide, halogens, amines, and acrylamide, to name a few. ${ }^{1,2}$ Collisional quenching has been used to reveal the rates of diffusion of quenchers in proteins ${ }^{3-6}$ and in membranes, ${ }^{7-8}$ and to determine the relative exposure of fluorophores in proteins and membranes to the aqueous phase or to the internal region of the macromolecules. ${ }^{8-11}$ An additional use of collisional quenching is to control the excited-state lifetime, and thereby alter the time available for rotational diffusion or spectral relaxation. ${ }^{12}$ Such lifetimeresolved measurements have been used to quantify rotational diffusion and time-dependent spectral shifts of labeled membranes ${ }^{13-14}$ and segmental motions of tryptophan residues in proteins. ${ }^{15-16}$ The occurrence of significant collisional quenching requires that the media or macromolecule be adequately fluid for translational motion during the excited-state lifetime and that the fluorophore be accessible to the quenchers. In addition, one must

\footnotetext{
Address all correspondence to Joseph R. Lackowicz. Fax: 410/706-8408. *Current affiliation: Technical University of Gdańsk, Faculty of Applied Physics and Mathematics, 80-952 Gdańsk, Poland.
}

consider the possibility that the quenchers interact with the macromolecules and thereby alter their properties. In spite of these limitations, fluorescence quenching is a powerful method for studying the structure and dynamics of biological macromolecules.

We now describe the use of light itself as a quencher of fluorescence. In recent reports we have shown that illumination of samples with wavelengths overlapping the emission spectra can result in quenching by the process of stimulated emission. Since the samples are observed 90 deg to the incident light, the observed intensity is decreased, and we refer to this phenomenon as light quenching. ${ }^{17}$ The extent of light quenching depends on overlap of the emission spectra with the quenching wavelength, the time delay between the excitation and quenching pulses, and on the orientation of the fluorophore relative to the electric vector of the quenching beam. ${ }^{18-21}$ The occurrence of light quenching with high repetition-rate ps dye lasers has been demonstrated by both steady-state ${ }^{18-21}$ and time-resolved measurements. ${ }^{22}$

In this article we show that wavelength-selective light quenching can be used to preferentially deplete the emission of one fluorophore in a mixture

1083-3668/97/\$10.00 (C) 1997 SPIE 


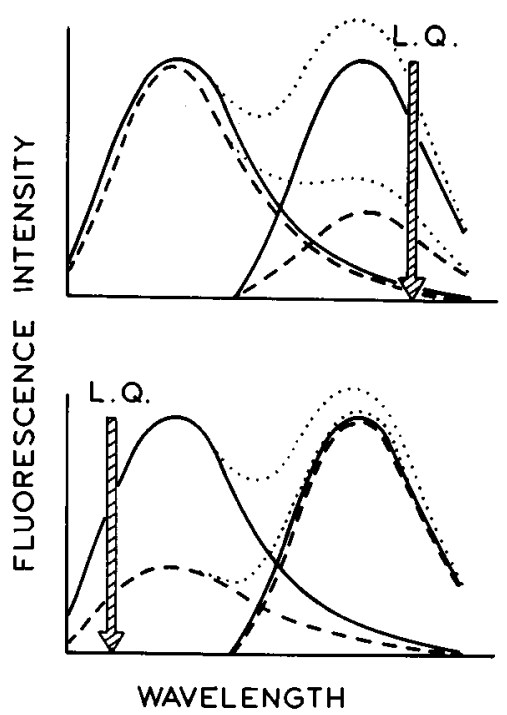

Fig. 1 Intuitive description of wavelength-selective light quenching. The solid and dashed lines show the emission spectra of particular components in the presence and absence of light quenching, respectively. The dotted lines illustrate the respective spectra emitted by the mixture of the components.

of fluorophores. The nature of these experiments is shown in Figure 1. Suppose the emission is due to two fluorophores, which emit at overlapping but different wavelengths. Illumination of the sample with long wavelengths, overlapping the emission of the longer wavelength fluorophores, is expected to preferentially quench this species. In this case long wavelength quenching is expected to result in a blue shift in the steady-state emission spectrum. Conversely, illumination on the short wavelength side of the emission is expected to quench the shorter wavelength fluorophore, resulting in a red shift in the emission spectrum. The possibility of wavelength-selective light quenching was demonstrated first for a mixture of fluorophores emitting at different wavelengths. We then determined that the emission of an aqueous phase fluorophore can be quenched in the presence of the same fluorophore bound to a protein. Finally we show preferential quenching of a buried tryptophan residue in the presence of a solvent-exposed tryptophan residue.

\section{THEORY}

We will consider two types of light quenching experiments. In a "one-beam" experiment, excitation and emission are due to the same light beam, and occur simultaneously during the excitation pulse. In this case the excitation and quenching wavelengths are identical. In a "two-beam" experiment, excitation and quenching are due to different light beams, which are typically of different wave- lengths. In this case the first pulse excites the sample, and the second pulse quenches the emission.

The theory for time-resolved measurements is somewhat different for one-beam and two-beam light quenching, and a complete description will be presented elsewhere. ${ }^{23}$ In the case of one-beam light quenching, the intensity decay of the twofluorophore mixture can be expressed as

$$
I(t)=J\left[\frac{f_{1}}{\tau_{1}} \exp \left(-t / \tau_{1}\right)+\frac{f_{2}}{\tau_{2}} \exp \left(-t / \tau_{2}\right)\right],
$$

where $\tau_{k}$ are the respective fluorescence lifetimes, $J$ is the steady-state emission intensity of the mixture, and $f_{k}$ are the fractions of fluorescence contributed by the fluorophores to the steady-state emission. In the case of two-beam light quenching, the intensity decay displays an instantaneous jump at time $t=t_{d}$ describing the time of the arrival of the quenching pulse. ${ }^{17,22}$ The intensity decreases may be different for each fluorophore and are described by parameters $q_{k}$

$$
q_{k}=\frac{I_{k b}-I_{k a}}{I_{k b}},
$$

where $I_{k b}$ and $I_{k a}$ are the fluorescence intensities of the respective fluorophores immediately before and after the quenching pulse. The expression for the intensity decay in the presence of two-beam light quenching takes the form

$$
=\left\{\begin{array}{c}
J\left[\frac{f_{1}}{Z_{1}} \exp \left(-t / \tau_{1}\right)+\frac{f_{2}}{Z_{2}} \exp \left(-t / \tau_{2}\right)\right], \quad 0 \leqslant t \leqslant t_{d} \\
J\left[\frac{f_{1}}{Z_{1}}\left(1-q_{1}\right) \exp \left(-t / \tau_{1}\right)+\frac{f_{2}}{Z_{2}}\left(1-q_{2}\right)\right. \\
\left.\quad \times \exp \left(-t / \tau_{2}\right)\right], \quad t>t_{d} .
\end{array}\right.
$$

where $Z_{k}$ are the normalization factors defined as

$$
Z_{k}=\tau_{k}\left[1-q_{k} \exp \left(-t_{d} / \tau_{k}\right)\right]
$$

We examined the intensity decays of the mixture, with and without light quenching, using the frequency-domain method. For the analysis, the frequency responses are calculated from Eqs. (1) and (3) at each modulation frequency $(\omega)$ using the two frequency-dependent quantities. ${ }^{24,25}$

$$
\begin{aligned}
& N_{\omega}=\int_{0}^{\infty} I(t) \sin (\omega t) \mathrm{d} t, \\
& D_{\omega}=\int_{0}^{\infty} I(t) \cos (\omega t) \mathrm{d} t .
\end{aligned}
$$


These quantities were then used to find the calculated phase angle $\left(\phi_{c \omega}\right)$ and the calculated luminescence modulation $\left(m_{c \omega}\right)$

$$
\begin{gathered}
\phi_{c \omega}=\arctan \left(N_{\omega} / D_{\omega}\right), \\
m_{c \omega}=\frac{1}{J}\left(N_{\omega}^{2}+D_{\omega}^{2}\right)^{1 / 2} .
\end{gathered}
$$

The best-fitted parameters, $f_{k}$ and $q_{k}$, and goodness of fit were determined by the minimum value of

$$
\chi_{R}^{2}=\frac{1}{\nu} \sum_{\omega}\left[\left(\frac{\phi_{\omega}-\phi_{c \omega}}{\delta \phi}\right)^{2}+\left(\frac{m_{\omega}-m_{c \omega}}{\delta m}\right)^{2}\right],
$$

where $\phi_{\omega}$ and $m_{\omega}$ are the experimental phase and modulation, respectively, $\delta \phi=0.2 \mathrm{deg}$ and $\delta m$ $=0.005$ are the experimental uncertainties, and $\nu$ is the number of degrees of freedom.

\section{MATERIAls AND Methods}

\subsection{ONE-BEAM LIGHT QUENCHING}

The experimental arrangements are different for one-beam and two-beam light quenching. We used one-beam light quenching to study selective quenching of tryptophan residues in RNase $T_{1}$ and Adrenocen Ticotropic hormone (ACTH). For the one-beam experiments, the excitation and light quenching are due to the same incident beam. In order to obtain overlap of the excitation with the emission spectrum, it is necessary to excite the sample on the extreme long wavelength edge of the absorption spectra. We used an experimental arrangement similar to that used previously for onebeam light quenching ${ }^{18}$ except that the light source was a frequency-doubled and cavity-dumped rhodamine $6 \mathrm{G}$ dye laser at $300 \mathrm{~nm}$. The dye laser was synchronously pumped by the 514-nm output of a mode-locked argon ion laser. The pulse width was near 5 ps, the repetition rate was $3.795 \mathrm{MHz}$, and the average power was $5 \mathrm{~mW}$. This light was focused to a spot size about $20 \mu \mathrm{m}$ in diameter, resulting in a maximum intensity of about $2 \times 10^{3}$ $\mathrm{W} / \mathrm{cm}^{2}$. Without focusing, the spot size was about 2 $\mathrm{mm}$ in diameter. The emission was selectively observed from the focal region of the illuminated sample using a spatial filter $3 \mathrm{~mm}$ high and $200 \mu \mathrm{m}$ wide.

The excitation was polarized vertically, as occurs from the output of our dye laser. The emission was observed through a Corning filter, which transmits light above $320 \mathrm{~nm}$. For intensity measurements, the emission polarizer was 54.7 deg from the vertical. Control measurements using the buffer without the proteins gave signals less than $0.5 \%$ of the protein emission, for all polarization conditions and excitation (quenching) wavelengths.

Ribonuclease $T_{1}$ was a gift from Dr. Maurice Eftink, University of Mississippi, and the ACTH was obtained from Ciba-Geigy. The proteins were dissolved in $0.1 \mathrm{M}$ sodium acetate, $\mathrm{pH}$ 5.5. For measurements of each protein alone, the concentrations were near $2 \times 10^{-5} \mathrm{M}$. In the mixture of RNase $T_{1}$ and $\mathrm{ACTH}$, the concentrations were 1.1 and $1.3 \times 10^{-5} M$, respectively.

\subsection{TWO-BEAM LIGHT QUENCHING}

Two-beam light quenching was used for studies of the DCS-Prodan mixture, and for light quenching of free and human serum albumin (HSA)-bound Prodan. The experimental arrangement for twobeam light quenching was the same as described previously. ${ }^{21,22}$ The light source was again the R6G dye laser, but in this case the frequency-doubled output at $285 \mathrm{~nm}$ was used for excitation, and the fundamental output at $570 \mathrm{~nm}$ was used for light quenching. The average power at 285 and $570 \mathrm{~nm}$ was 5 and $150 \mathrm{~mW}$, respectively. The region of overlap of the excitation and quenching beams was selectively observed with the $3 \mathrm{~mm} \times 200 \mu \mathrm{m}$ spatial filter. The time delay between the excitation and quenching pulses was 20 ps for all experiments.

In the mixture of DCS and Prodan, the concentrations in the dimethylformamide (DMF) solvent were $2 \times 10^{-5} \mathrm{M}$ and $3 \times 10^{-5} \mathrm{M}$, respectively. The human serum albumin (HSA) was in $20 \mathrm{mM}$ phosphate buffer, $\mathrm{pH} 7.4$, with an HSA concentration near $10^{-5} \mathrm{M}$ and a Prodan concentration of $6 \times 10^{-5}$ $M$.

Intensity and anisotropy decay measurements were obtained using frequency domain (FD) instrumentation described previously. ${ }^{26-27}$ For intensity decay measurements, the excitation was vertically polarized and the emission observed through a polarizer $54.7 \mathrm{deg}$ from the vertical. The FD intensity decay data were analyzed as described previously. $^{24-25}$

\section{RESUlts}

\subsection{TWO-BEAM LIGHT QUENCHING OF A FLUOROPHORE MIXTURE}

To evaluate the prospects for wavelength-selective light quenching, we first examined a mixture of two fluorophores, DCS and Prodan. These fluorophores were selected for the possibility of excitation at the same wavelength $285 \mathrm{~nm}$, and for different fractional overlaps of their emission spectra with the 570-nm quenching wavelength (Figure 2). In the DMF solvent, Prodan and DCS display emission maxima of 445 and $515 \mathrm{~nm}$, respectively. The intensity decay of each fluorophore alone is nearly a single exponential, with decay times of 0.68 and 3.9 ns, respectively (not shown).

Examination of Figure 2 indicates that the emission spectrum of DCS overlaps more strongly with the quenching beam than does the emission of Prodan. Hence, one expects selectively more light quenching of DCS than Prodan for 570-nm quenching. The emission spectrum of the mixture was 


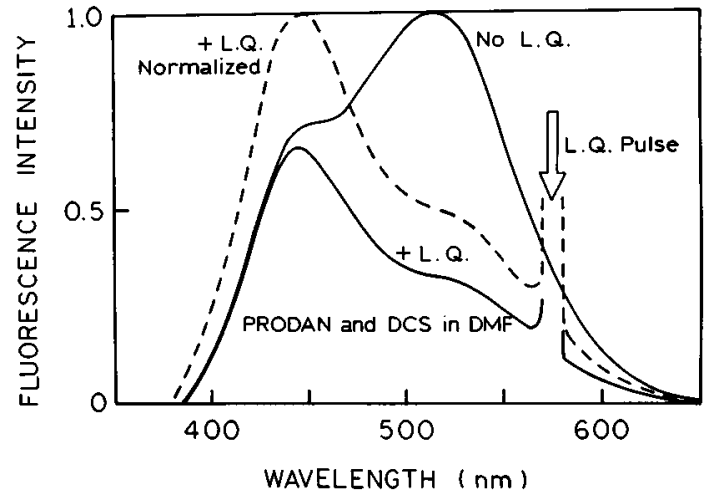

Fig. 2 Emission spectra of a mixture of Prodan and DCS in DMF.

found to be strongly blue shifted upon 570-nm illumination (Fig. 2, $+L Q$ and dashed line). This effect was immediately reversible upon blocking of the quenching beam.

We also determined the extent of light quenching from the intensity decays measured in the frequency domain. Intensity decays were measured at $480 \mathrm{~nm}$, where we expected comparable intensities from each fluorophore. In the absence of light quenching, the intensity decay is a double exponential [Figure 3(a), and Table 1], as is expected for a mixture of two fluorophores, each of which displays a single exponential intensity decay. In the presence of light quenching, the intensity decay remains a double exponential. However, the shape of the frequency response is altered, as can be seen from the comparison of the data with and without light quenching in Fig. 3(b). Analysis of these data indicates that the decay times were unchanged by light quenching, but the fractional intensity of each fluorophore was altered by light quenching. To be specific, the intensity of DCS was quenched by $68.5 \%$, while the intensity of Prodan was only quenched by $3.4 \%$ (Table 1 ). Without light quenching, the fractional intensities of DCS and Prodan are 0.75 and 0.25 , respectively. With light quenching, the respective fractional intensities are nearly equal- 0.51 and 0.49 . The higher fractional contri-

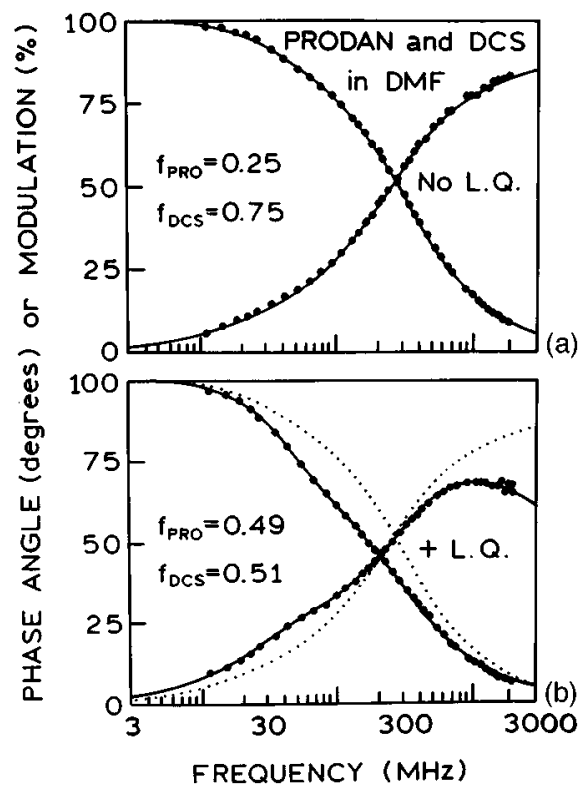

Fig. 3 Frequency-domain intensity decay of a mixture of Prodan and DCS in (a) the absence and (b) the presence of light quenching. The solid lines show the best fit to the experimental data (0) based on Eq. (3) for $\tau_{1} \equiv \tau_{\mathrm{DCS}}=0.68 \mathrm{~ns}$ and $\tau_{2} \equiv \tau_{\mathrm{PRO}}=3.9 \mathrm{~ns}$. The dotted line represents the frequency response in the absence of light quenching (shown by the solid line in the upper panel).

bution of thelonger lifetime Prodan can be seen in the FD data by the higher phase angles and lower modulation values from 30 to $100 \mathrm{MHz}$. These data show that the selectivity of light quenching can be quite high, in this case DCS being quenched 20-fold more strongly than Prodan.

In a previous report we described oscillations in the frequency-domain data due to time-delayed light quenching. ${ }^{22}$ Hence, one may question the absence of oscillations in Fig. 3. The oscillations are not visually apparent because of the short decay time of 20 ps. The oscillations are just becoming apparent in the data above $1 \mathrm{GHz}$ (Figure 3).

Table 1 Intensity decays in the absence and presence of long-wavelength two-pulse light quenching.

\begin{tabular}{lcccccc}
\hline Sample & Quenching & $f_{1}$ & $f_{2}$ & $q_{1}$ & $q_{2}$ & $\chi_{R}^{2}$ \\
\hline $\begin{array}{c}\text { DCS }\left(\tau_{1}=0.68 \mathrm{~ns}\right) \\
\text { and Prodan }\end{array}$ & No LQ & 0.749 & 0.251 & 0 & 0 & 1.2 \\
$\begin{array}{c}\left(\tau_{2}=3.9 \mathrm{~ns}\right) \text { in DMF } \\
\begin{array}{l}\text { Prodan }\left(\tau_{1}=0.57 \mathrm{~ns}\right) \\
\text { and Prodan-HSA }\end{array}\end{array}$ & $+\mathrm{LQ}$ & 0.506 & 0.494 & 0.685 & 0.034 & 1.2 \\
$\left(\tau_{2}=3.7 \mathrm{~ns}\right)$ & No LQ & 0.858 & 0.142 & 0 & 0 & 1.3 \\
\hline
\end{tabular}

Note: Intensity decays were measured at $480 \mathrm{~nm}$. Values of the lifetimes $\tau_{1}$ and $\tau_{2}$ are those used in Eq. (3) and refer to both the absence and presence of light quenching. These lifetimes were held fixed during the analysis. 


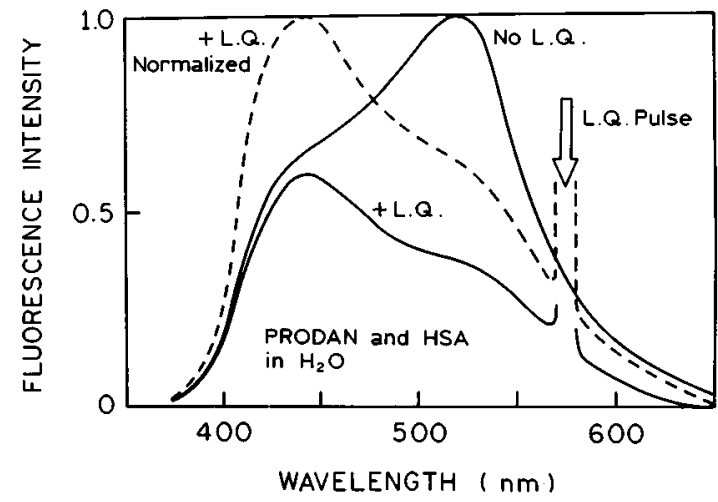

Fig. 4 Emission spectra of Prodan partially bound to HSA.

\subsection{TWO-BEAM LIGHT QUENCHING OF PRODAN IN SOLUTION AND BOUND TO HSA}

We next considered the possibility of selective light quenching of the same fluorophore, Prodan, in two different environments. In water, Prodan displays a red-shift emission with a maximum near $510 \mathrm{~nm}$ (Figure 4). Upon binding to HSA, the emission of Prodan is strongly blue shifted to an emission maximum near $445 \mathrm{~nm}$. Based on these spectral properties, we expected free Prodan to be more strongly quenched by $570-\mathrm{nm}$ illumination.

Emission spectra of Prodan partially bound to HSA are shown in Figure 4. Upon illumination with $570 \mathrm{~nm}$, the emission spectrum is strongly blue shifted, which is consistent with preferential quenching of free Prodan. We also measured the frequency-domain intensity decay of Prodan. In water, and when completely bound the HSA, the intensity decays are reasonably single exponentials with decay times of 0.57 and 3.7 ns, respectively (not shown). When Prodan is partially bound to HSA, the intensity decay of the solution is a double exponential (Figure 5 and Table 1). In the absence of light quenching, the time-resolved emission is mostly due to free Prodan, which displayed a fractional intensity of 0.858 . Upon $570-\mathrm{nm}$ light quenching, the fractional intensity of free Prodan decreased to 0.664 (Table 1). The higher contribution of HSA-bound Prodan to the time-resolved emission can be seen from the increase in phase angle and decrease in modulation near $50 \mathrm{MHz}$ (Figure 5). The decrease in phase angle above $500 \mathrm{MHz}$ in the presence of light quenching (Figure 5) is due to the onset of oscillations in the FD data caused by the time-delayed quenching pulse. ${ }^{22}$

\subsection{ONE-BEAM LIGHT QUENCHING OF THE BURIED TRYPTOPHAN RESIDUE IN RIBONUCLEASE $T_{1}$}

In the previous section we demonstrated that light quenching could preferentially quench a fluorophore in the aqueous phase, which is also possible

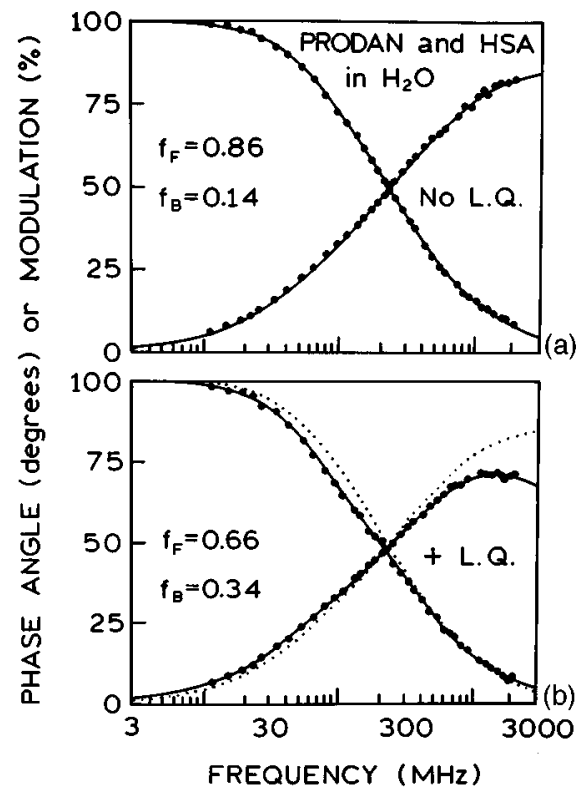

Fig. 5 Frequency-domain intensity decay of Prodan-HSA, in (a) the absence and (b) the presence of light quenching. The solid lines show the best fit to the experimental data (0) based on Eq. (3) for $\tau_{1} \equiv \tau_{F}=0.57 \mathrm{~ns}$ and $\tau_{2} \equiv \tau_{B}=n 3.7 \mathrm{~ns}$. The dotted line represents the frequency response in the absence of light quenching (shown by the solid line in the upper panel).

for collisional quenchers. Hence we questioned whether light quenching could preferentially quench a fluorophore shielded from the solvent. To answer this question, we examined a mixture of two proteins, ACTH and RNase $T_{1}$, each of which contains a single tryptophan residue. The residue in ACTH is fully exposed to solvent, ${ }^{1,28}$ and displays a red-shifted emission [Figure 6(a)]. The tryptophan residue in RNase $T_{1}$ is completely shielded from the solvent, ${ }^{28-29}$ and displays a blue-shifted emission (Figure 6).

Because of limitations of our laser sources, we were not able to accomplish two-beam light quenching of proteins with a time-delayed pulse. Hence, we use one-beam light quenching in which the focused excitation pulse both excites the fluorophores and causes light quenching. Excitation and quenching were accomplished using 300-nm excitation, which overlaps with the emission of RNase $T_{1}$ but not with the emission of ACTH. In the mixture of RNase $T_{1}$ and ACTH without light quenching, the emission spectrum shows a dominant contribution of RNase $T_{1}$ [Figure 6(b)]. In the presence of light quenching, the emission maximum shifts to longer wavelengths, demonstrating that the buried tryptophan in RNase $T_{1}$ is quenched more strongly than the exposed residue in ACTH. Preferential quenching of a buried residue is the opposite of that usually observed with collisional quenchers, which illustrates the complementary characteristics of light quenching and collisional quenching. 

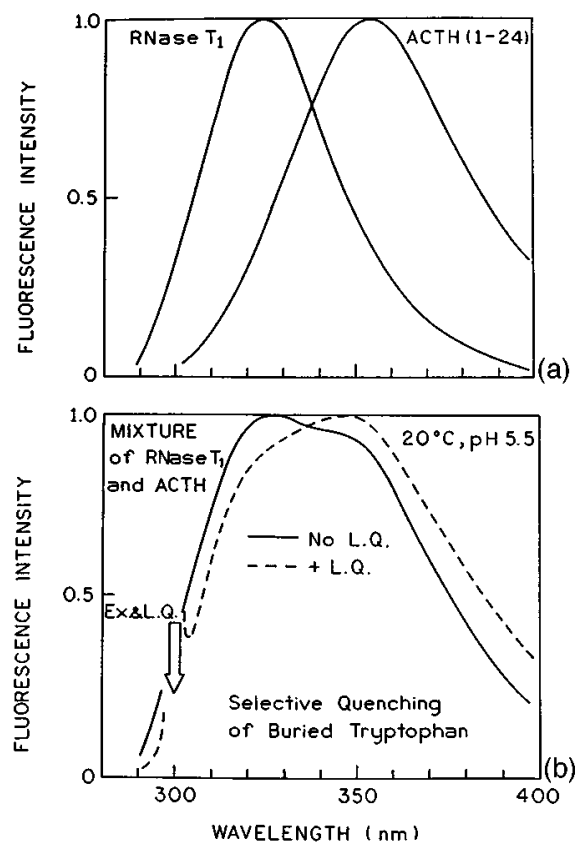

Fig. 6 Emission spectra of (a) RNase $T_{1}$ and $A C T H$ and (b) a mixture of RNase $T_{1}$ and ACTH with $(---)$ and without $(-)$ light quenching.

We also examined how the intensity decays of the protein mixture have affected by light quenching. The intensity decay of RNase $T_{1}$ alone is a single exponential of $3.85 \mathrm{~ns}^{28-29}$ The intensity decay of ACTH is not a single exponential, and requires at least three decay times to describe its intensity decay. ${ }^{29}$ However, for simplicity we treated the intensity decay of ACTH as a single exponential with a mean decay time of 2.36 ns. Frequency-domain intensity decay data for a mixture of RNase $T_{1}$ and $\mathrm{ACTH}$ are shown in Figure 7. The intensity decay in the mixture could not be fit to the single exponential model (Table 2). However, the double exponential model gave a reasonably good fit. Without light quenching, the double exponential analysis

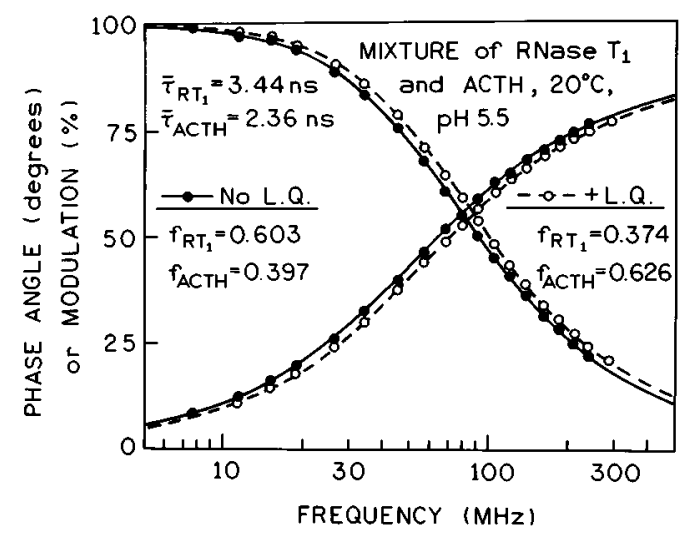

Fig. 7 Frequency response of a mixture of RNase $T_{1}$ and $A C T H$ without (- -) and with (- $\bigcirc--)$ one-beam light quenching.
Table 2 Intensity decays of ACTH and RNase $T_{1}$, in the absence and presence of short-wavelength one-pulse light quenching.

\begin{tabular}{|c|c|c|c|c|}
\hline Sample & Quenching & $f_{1}$ & $f_{2}$ & $\chi_{R}^{2}$ \\
\hline RNase $T_{1}\left(\tau_{1}=3.44\right)$ & No LQ & 1.0 & - & 2.1 \\
\hline $\mathrm{ACTH}\left(\tau_{2}=2.36\right)$ & No $L Q$ & - & 1.0 & 79.4 \\
\hline RNase $T_{1}\left(\tau_{1}=3.44\right)$ and & No $L Q$ & 1.0 & - & $7.2^{a}$ \\
\hline \multirow[t]{2}{*}{$\mathrm{ACTH}\left(\tau_{2}=2.36\right)$} & No $L Q$ & 0.60 & 0.40 & 2.1 \\
\hline & $+L Q$ & 0.37 & 0.63 & 2.1 \\
\hline
\end{tabular}

yields decay times of 3.44 and $2.36 \mathrm{~ns}$ with fractional intensities of $f_{1}=0.603$ and $f_{2}=0.397$ for RNase $T_{1}$ and $\mathrm{ACTH}$, respectively (Table 2). As predicted from the emission spectrum, the emission of RNase $T_{1}$ is dominant without light quenching. In the presence of light quenching, the frequency response of the mixture shifts to higher frequencies, indicating an increased contribution of the ACTH. Light quenching occurs preferentially for RNase $T_{1}$, so that the emission of ACTH becomes the major component in the intensity decay in the presence of light quenching $\left(f_{2}=0.625\right)$. These results demonstrate the possibility of wavelength-selective light quenching of tryptophan residues in proteins.

\section{DISCUSSION}

What are the advantages of using light as a quencher, compared with the usual use of collisional quenchers added to the solution of macromolecules. ${ }^{2}$ One obvious advantage is the immediate reversal of quenching by blocking or defocusing the incident beam. Small amounts of light quenching can thus be detected using an optical chopper and lock-in detection methods. In contrast, collisional quenchers must be removed by dialysis or chromatographic methods.

An additional advantage of light quenching is that it can occur in highly viscous solutions that do not allow significant translational diffusion during the excited-state lifetime. Since light quenching depends on spectral overlap, and not physical contact between the fluorophore and quencher, light quenching can be expected to occur for fluorophores that are not accessible to collisional quenchers, as was shown for RNase $T_{1}$. Depending on the emission spectra and quenching wavelength, light quenching can occur for either solvent-exposed or for buried residues. In contrast, significant collisional quenching only occurs in low-viscosity conditions, and requires molecular contact between the fluorophore and quencher. 
Light quenching also depends on the lifetime of the excited state. If the solution contains two fluorophores with different decay times, the extent of quenching of each fluorophore depends on its excited state population upon arrival of the quenching pulse. This property of light quenching may be especially valuable in studies of multichromophore systems, such as photosynthetic reaction centers or phycobiliproteins, ${ }^{30-32}$ in which energy is transferred between several chromophores. Examination of the emission properties with various time delays may provide information about the energy transfer process.

It should also be noted that light quenching is not expected to be photodestructive. Since light quenching returns the fluorophores to the ground state more rapidly than expected for the spontaneous decay, one can expect the fluorophore to be protected from photochemical processes by light quenching. Such protection against photochemical degradation has been used as a basis for measuring energy transfer efficiencies in fluorescence microscopy. ${ }^{33-34}$

Finally, light quenching differs from collisional quenching in that light quenching depends on the orientation of the fluorophore relative to the polarized quenching beam. In contrast, there is no known dependence of collisional quenching on orientations. These differences between collisional quenching and light quenching indicate that the methods are complementary, and in combination can provide additional information about biological macromolecules.

\section{Acknowledgment}

This work was supported by the National Institute of Health National Center for Research Resources, grants RR-08119 and RR-10416.

\section{REFERENCES}

1. M. R. Eftink, "Fluorescence quenching: theory and applications," in Topics in Fluorescence Spectroscopy, Vol. 2, Principles, pp. 53-126, Plenum Press, New York (1991).

2. J. R. Lakowicz (Ed.), Principles of Fluorescence Spectroscopy, Plenum Press, New York (1983).

3. J. R. Lakowicz and G. Weber, "Quenching of protein fluorescence by oxygen. Detection of structural fluctuations in proteins on the nanosecond timescale," Biochemistry 12, 4171-4179 (1973).

4. D. B. Calhoun, J. M. Vanderkooi, and S. W. Englander, "Penetration of small molecules into proteins studied by quenching of phosphorescence and fluorescence," Biochemistry 22, 1533-1539 (1983).

5. D. B. Calhoun, J. M. Vanderkooi, G. V. Woodrow, and S. W. Englander, "Penetration of dioxygen into proteins studied by quenching of phosphorescence and fluorescence," Biochemistry 22, 1526-1532 (1983).

6. D. B. Calhoun, S. W. Englander, W. W. Wright, and J. M. Vanderkooi, "Quenching of room temperature protein phosphorescence by added small molecules," Biochemistry 27, 8466-8476 (1988).

7. M. F. Blackwell, K. Gounaris, S. J. Zara, and J. Barber, "A method for estimating lateral diffusion coefficients in membranes from steady-state fluorescence quenching studies,' Biophys. J. 51, 735-744 (1987).
8. M. Ollmann, G. Schwarzmann, K. Sandhoff, and H.-J. Galla, "Pyrene-labeled gangliosides: micelle formation in aqueous solution, lateral diffusion, and thermotropic behavior in phosphatidylcholine bilayers," Biochemistry 26, 5943-5952 (1987).

9. S. S. Lehrer, "Solute perturbation of protein fluorescence. The quenching of the tryptophan fluorescence of model compounds and of lysozyme and iodide ion," Biochemistry 10, 3254-3263 (1971).

10. E. Blatt and W. H. Saywer, "Depth-dependent fluorescent quenching in micelles and membranes," Biochim. Biophys. Acta 822, 43-62 (1985).

11. T. Markello, A. Zlotnick, J. Everett, J. Tennyson, and P. W. Holloway, "Determination of the topography of cytochrome $b_{5}$ in lipid vesicles by fluorescence quenching," Biochemistry 24, 2895-2901 (1985).

12. J. R. Lakowicz, "Fluorescence spectroscopic investigations of the dynamic properties of proteins, membranes and nucleic acids," J. Biochem. Biohys. Meth. 2, 90-119 (1986).

13. J. R. Lakowicz, F. G. Prendergast, and D. Hogen, "Fluorescence anisotropy measurements under oxygen quenching conditions as a method to quantify the depolarizing rotations of fluorophores: application to diphenylhexatriene in solvents and in lipid bilayers," Biochemistry 18, 520-527 (1979).

14. J. R. Lakowicz and D. Hogen, "Dynamic properties of the lipid-water interface region of model membranes as revealed by lifetime-resolved fluorescence emission spectra," Biochemistry 20, 1366-1373 (1981).

15. J. R. Lakowicz, B. Maliwal, H. Cherek, and A. Balter, "Rotational freedom of tryptophan residues in proteins and peptides quantified by lifetime-resolved fluorescence anisotropies," Biochemistry 22, 1741-1752 (1983).

16. Z. Lakos, A. Szarka, L. Koszorus, and B. Somogyi "Quenching-resolved emission anisotropy: a steady state fluorescence method to study protein dynamics," J. Photochem. Photobiol. 27, 55-60 (1995).

17. J. R. Lakowicz, I. Gryczynski, J. Kuśba, and V. Bogdanov, "Light quenching of fluorescence: a new method to control the excited state lifetime and orientation of fluorophores," Photochem. Photobiol. 60, 546-562 (1994).

18. J. R. Lakowicz, I. Gryczynski, V. Bogdanov, and J. Kusba, "Light quenching and fluorescence depolarization of rhodamine B," J. Phys. Chem. 98, 334-342 (1994).

19. I. Gryczynski, V. Bogdanov, and J. R. Lakowicz, "Light quenching of tetraphenylbutadiene fluorescence observed during two-photon excitation," J. Fluoresc. 3, 85-92 (1993).

20. J. Kuśba, V. Bogdanov, I. Gryczynski, and J. R. Lakowicz, "Theory of light quenching: effects on fluorescence polarization, intensity and anisotropy decays," Biophys. J. 67, 20242040 (1994).

21. I. Gryczynski, J. Kuśba, Z. Gryczynski, H. Malak, and J. R. Lakowicz, "Effect of fluorescence quenching by stimulated emission on the spectral properties of a solvent-sensitive fluorophore, "'J. Phys. Chem. 100 (24), 10135-10144 (1996).

22. I. Gryczynski, J. Kuśba, and J. R. Lakowicz, "Light quenching and depolarization of fluorescence observed with laser pulses as observed by frequency-domain fluorometry," $\mathrm{J}$. Phys. Chem. 98, 8886-8895 (1994).

23. I. Gryczynski, J. Kuśba, and J. R. Lakowicz, "Selective light quenching in mixtures of fluorophores J. Fluoresc. (submitted, 1996).

24. E. Gratton, J. R. Lakowicz, B. Maliwal, H. Cherek, G. Laczko, and M. Limkeman, "Resolution of mixtures of fluorophores using variable-frequency phase and modulation data," Biophys. J. 46, 479-486 (1984).

25. J. R. Lakowicz, E. Gratton, G. Laczko, H. Cherek, and M. Limkeman, "Analysis of fluorescence decay kinetics from variable-frequency phase shift and modulation data," Biophys. J. 46, 463-477 (1984).

26. J. R. Lakowicz, and B. P. Maliwal, "Construction and performance of a variable-frequency phase-modulation fluorometer," Biophys. Chem. 21, 61-78 (1985).

27. G. Laczko, J. R. Lakowicz, I. Gryczynski, Z. Gryczynski, and H. Malak, "A $10 \mathrm{GHz}$ frequency-domain fluorometer," Rev. Sci. Instrum. 61, 2331-2337 (1990). 
28. M. R. Eftink, and C. A. Ghiron, "Frequency domain measurements of the fluorescence lifetime of ribonuclease $T_{1}$, " Biophys. J. 52, 467-473 (1987).

29. J. R. Lakowicz, I. Gryczynski, H. Szmacinski, H. Cherek, and N. Joshi, "Anisotropy decays of single tryptophan proteins measured by $\mathrm{GHz}$ frequency-domain fluorometry with collisional quenching," Eur. Biophys. J. 19, 125-140 (1991).

30. J. C. White, and L. Stryer, "Photostability studies of phycobiliprotein fluorescence labels," Anal. Biochem. 161, 442-452 (1987).

31. A. R. Holzwarth, J. Wendler, and W. Wehremeyer, "Studies on chromophore coupling in isolated phycobiliproteins. I. Picosecond fluorescence kinetics of energy transfer in phy- -cocyanin 645 from Chroomonas sp.," Biochem. Biophys. Acta. 934, 275-281 (1983).

32. R. MacColl, D. Guard-Friar, T. J. Ryan, K. Casatorday, and $\mathrm{P}$. Wu, "The route of exciton migration in phycocyanin 612," Biochem. Biophys. Acta 934, 275-281 (1988).

33. T. M. Jovin, G. Marriott, R. M. Clegg, and D. J. Arndt-Jovin, "Photophysical processes exploited in digital imaging microscopy: fluorescence resonance energy transfer and delayed luminescence," Ber. Gunsenges. Phys. Chem. 93, 387391 (1989).

34. U. Kubitscheck, M. Kircheis, R. Schweitzer-Stenner, W. Dreybrodt, T. M. Jovin, and I. Pecht, "Fluorescence resonance energy transfer on single living cells," Biophys. J. 60, 307-318 (1991). 